\title{
Characterization of the nuclear localization signals of duck circovirus replication proteins
}

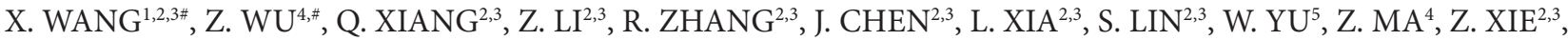 \\ S. JIANG ${ }^{2,3^{*}}$
}

\begin{abstract}
${ }^{1}$ College of Life Science, Linyi University, Shuangling Road, Linyi City, Shandong 276005, P. R. China; ${ }^{2}$ Department of Preventive Veterinary Medicine, College of Veterinary Medicine, Shandong Agricultural University, Taian, Shandong 271018, P. R. China; ${ }^{3}$ Shandong Provincial Key Laboratory of Animal Biotechnology and Disease Control and Prevention, Taian, Shandong 271018, P. R. China; ${ }^{4}$ Shanghai Veterinary Research Institute, Chinese Academy of Agricultural Sciences, No. 518, Ziyue Road, Shanghai, 200241, P. R. China; ${ }^{5}$ Department of Basic Medical Sciences, Taishan Medical College, Taian, Shandong 271000, P. R. China
\end{abstract}

Received March 24, 2015; revised July 22, 2015; accepted October 22, 2015

\begin{abstract}
Summary. - Duck circovirus (DuCV) possess a circular, single-stranded DNA genome that requires the replication protein (Rep) for its replication. Based on the viral genotype, there are two categories of Rep proteins: Rep1 and Rep2. To characterize the nuclear localization signals (NLSs) conferring the nuclear localization of the Rep proteins, defined coding regions of the rep gene of two genotypes of DuCV were cloned and co-expressed with the red fluorescent protein DsRed2. The results showed that deleting the putative $\mathrm{N}$-terminal NLS located at amino acid residues 10-37 of Rep1 and Rep2 abrogated nuclear translocation, while deleting the putative C-terminal NLS located at residues 244-274 of Rep1 did not significantly alter its subcellular localization, confirming that only the NLS located at residues 10-37 in the N-termini of the Rep proteins had nuclear targeting activity.
\end{abstract}

Keywords: duck circovirus; genotype; Rep protein; nuclear localization signal

Duck circovirus (DuCV) is a member of the genus Circovirus of the Circoviridae family (Hattermann et al., 2003). Infection with DuCV is characterized by feathering disorders, poor body condition, and low body weight (Hattermann et al., 2003), and it results in multiple local lesions in the spleen, thymus, and the bursa of Fabricius in ducks (Soike et al., 2004; Liu et al., 2010a). Our previous studies provided evidence of possible horizontal and vertical transmission of DuCV and the simultaneous transmission of DuCV-1 and DuCV-2 from breeder ducks to ducklings (Liu et al., 2010a, 2014). Surveys showed that there was a high prevalence of DuCV in duck flocks of different species (Hattermann et al.,

*Corresponding author. E-mail: sjjiang@sdau.edu.cn; phone: +86 538 8245799. " These authors contributed equally to this work. Abbreviations: $\mathrm{CP}=$ capsid protein; $\mathrm{DuCV}=$ duck circovirus; $\mathrm{NLS}(\mathrm{s})=$ nuclear localization $\operatorname{signal}(\mathrm{s}) ; \mathrm{ORF}(\mathrm{s})=$ open reading frame(s); PCV = porcine circovirus; Rep = replication protein
2003; Soike et al., 2004; Jiang et al., 2008; Zhang et al., 2009; Liu et al., 2010a).

With a single-stranded circular genomic DNA of about $1.99 \mathrm{~kb}, \mathrm{DuCV}$ was classified into two genotypes, DuCV-1 and DuCV-2 (Wang et al., 2011; Zhang et al., 2012). Both DuCV-1 and DuCV-2 have similar genomic organizations, with three major ambisense open reading frames (ORFs) (Zhang et al., 2012). ORF1 (rep) is located on the viral plus-strand, and it encodes the viral replication-associated protein (Rep) (Hattermann et al., 2003). Located on the complementary strand, ORF2 (cap) encodes the immunogenic capsid protein, while ORF3 encodes the ORF3 protein, which has pro-apoptotic activity (Hattermann et al., 2003; Xiang et al., 2012).

Although circoviruses lack an autonomous DNA polymerase and are dependent on the replication machinery of the host cell for de novo DNA synthesis, Rep is required to initialize viral replication (Tischer et al., 1987; Meerts et al., 2005). In the case of porcine circovirus (PCV), ORF1 encodes, via 
differential splicing, two viral replication-associated proteins, Rep and Rep', (Mankertz and Hillenbrand, 2001; Mankertz et al., 2003; Cheung, 2003, 2004). Rep and Rep' are responsible for viral replication, and the replication of the PCV genome requires interactions between the Rep complex (Rep-Rep') and the cis-acting element at the origin of replication (Ori) that is present in the minimum-binding-site recognition region (Mankertz and Hillenbrand, 2001; Steinfeldt et al., 2001, 2006; Cheung, 2004, 2006, 2007).

Previous studies have revealed that the nuclear accumulation of the PCV1 and PCV2 capsid proteins is mediated by their distinct nuclear localization signal (NLS) motifs (Liu et al., 2001; Shuai et al., 2008). For PCV1, of the three potential NLSs that are located in the identical N-termini of Rep and Rep', NLS1 and NLS2 are required to mediate nuclear import, whereas NLS3 enhances the nuclear accumulation of the Rep proteins (Finsterbusch et al., 2005). The nuclear localization of the capsid protein (CP) of the beak feather disease virus (BFDV) was shown to be directed by three partially overlapping, bipartite NLSs, whereas Rep that was expressed on its own in insect cells was restricted to the cytoplasm. When co-expressed with the CP, Rep localized to the nucleus, strongly suggesting that an interaction with the CP facilitates the translocation of Rep into the nucleus (Heath et al., 2006).

Based on the phylogenetic analysis of complete amino acid sequences, the Rep protein of DuCV has been classified into two types: Rep1 and Rep2, respectively (Zhang et al., 2012). Our previous study showed that two independent, bipartite NLSs were located in the N-terminus of the CP at amino acid residues 1-17 and 18-36 (Xiang et al., 2013). Therefore, in this study, we aimed to determine whether the putative NLSs in Rep1 and Rep2 are required for their nuclear localization.

Using the online computer program cNLS Mapper (http:// nls-mapper.iab.keio.ac.jp/cgi-bin/NLS_Mapper_form.cgi), the Rep of DuCV-1 (Rep1) and the Rep of DuCV-2 (Rep2) were analyzed, and two potential NLSs were found at residues 10-37 and 244-274 of Rep1, whereas only a single NLS was found at residues 10-37 of Rep2 (Fig. 1). To test whether these potential NLSs were functional, we constructed a series of plasmids that expressed truncated Reps, which lacked the putative NLSs, fused with the red fluorescent protein DsRed2.

Two plasmids, pDuCV-1, which includes the complete genomic DNA of the DuCV-1 strain FJ0601 (EF370476) (Jiang et al., 2008), and pDuCV-2, which includes the complete genomic DNA of the DuCV-2 strain WF0701 (EU022375) (Zhang et al., 2012), were constructed and stored in our laboratories. Using plasmid $\mathrm{pDuCV}-1$ as template, the complete rep1 gene was amplified by PCR with primers Rep1-F and Rep1-R (Table 1). The entire rep2 gene was amplified with primers Rep2-F and Rep2-R using plasmid pDuCV-2 as a template. The PCR products were digested with EcoRI and BamHI (TaKaRa, Dalian, China) and inserted into the corre-

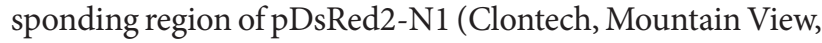
CA, USA). The recombinant plasmids, named pDs-Rep1 and pDs-Rep2, were used to express Rep fusion proteins tagged with the red fluorescent protein DsRed2.

Using pDs-Rep1 and pDs-Rep2 plasmid DNA as a template, four pairs of mutagenic primers were designed to construct the recombinant plasmids by the site directed mutagenesis method (Table 1). PCR was carried out in a $50 \mu \mathrm{l}$ volume with $100 \mathrm{ng}$ template plasmid DNA, $0.2 \mathrm{mmol} / \mathrm{l}$ (each) mutagenic primer, $200 \mu \mathrm{mol} / \mathrm{l}$ (each) deoxynucleoside triphosphates, $5 \mu$ l of $10 \times P f u$ PCR buffer (containing Mg2+), and $2.5 \mathrm{U}$ of cloned $P f u$ DNA polymerase (Stratagene, La Jolla, CA, USA). The amplification parameters consisted of an initial 5 min denaturation at $95^{\circ} \mathrm{C}$, followed by 18 cycles of $1 \mathrm{~min}$ at $95^{\circ} \mathrm{C}, 1 \mathrm{~min}$ at $60^{\circ} \mathrm{C}$, and an extension step at $68^{\circ} \mathrm{C}$, where the extension time in minutes was twice the length of the plasmid in kilobases, and a final 15 min extension step at $72^{\circ} \mathrm{C}$. After checking the DNA quality by agarose gel electrophoresis, the PCR products were digested with ten units of DpnI (TaKaRa) at $37^{\circ} \mathrm{C}$ for $1-2 \mathrm{hr}$. Then, $1-2 \mu \mathrm{l}$ of the DpnI-treated DNA was used to transform Escherichia coli DH5a competent cells (Li et al., 2008). A series of recombinant plasmids, pDs-Rep1 $\Delta 10-37$, pDs-Rep1 $\Delta 244-274$, pDs-Rep1 $\Delta 10-37 \Delta 244-274$, and pDs-Rep2 $\Delta 10-37$ were constructed and verified by sequence analysis.

The human non-small-cell lung carcinoma cell line H1299 (ATCC CRL-5803) was obtained from the American Type Culture Collection (Rockville, MD, USA). In a six-well culture plate, H1299 cells were cultured in Dulbecco's modified Eagle's medium (GIBCO BRL, Grand Island, NY, USA) supplemented with $10 \%$ fetal bovine serum (GIBCO BRL), $100 \mathrm{U} / \mathrm{ml}$ penicillin, and $100 \mu \mathrm{g} / \mathrm{ml}$ streptomycin. Cells were maintained at $37^{\circ} \mathrm{C}$ in a humidified $5 \% \mathrm{CO}_{2}$ incubator. When the cells reached approximately $70 \%$ confluence, they were washed twice with phosphate-buffered saline $(8.1 \mathrm{mmol} / \mathrm{l}$ $\mathrm{Na}_{2} \mathrm{HPO}_{4}, 1.5 \mathrm{mmol} / \mathrm{l} \mathrm{K \textrm {K } _ { 2 }} \mathrm{PO}_{4}, 140 \mathrm{mmol} / \mathrm{l} \mathrm{NaCl}$, and 3.0 $\mathrm{mmol} / \mathrm{l} \mathrm{KC1}, \mathrm{pH} 7.2)$ and transfected with $2 \mu \mathrm{g}$ of purified recombinant plasmids (pDs-Rep1, pDs-Rep1 $\Delta$ N10-37, pDs-Rep1 $\Delta$ N244-274, pDs-Rep1 $\Delta$ N10-37 $\Delta$ N244-274, pDs-Rep2, or pDs-Rep2 $\Delta$ N10-37) per well using the Xfect transfection reagent (Clontech) according to the manufacturer's protocol; H1299 cells transfected with an equal amount of the pDsRed2-N1 vector were used as negative controls.

DuCV-1-positive sera and DuCV-2-positive sera, which were the mixtures of serum samples from ten DuCV-1positive ducks and ten DuCV-2-positive ducks (Liu et al., 2010b), were stored in our laboratories. The H1299 cells were harvested $48 \mathrm{hr}$ post-transfection and analyzed by sodium dodecyl sulfate-polyacrylamide gel electrophoresis and western blotting as previously described (Xiang et al., 
2013). DuCV-positive sera were used at a 1:100 dilution; anti-glyceraldehyde 3-phosphate dehydrogenase (GAPDH) mouse monoclonal antibody (ProteinTech Group, Chicago, IL, USA) was used at a 1:2,000 dilution; anti-DsRed2 mouse monoclonal antibody (Santa Cruz Biotechnology, Dallas, TX, USA) was used at a 1:200 dilution; and horseradish peroxidase (HRP)-labeled goat anti-duck antibody (KPL, Gaithersburg, MD, USA) and HRP-labeled goat-anti-mouse IgG (Santa Cruz Biotechnology) were used at a 1:10,000 dilution. All constructs expressed proteins that corresponded to their expected molecular sizes, and no protein was detected in H1299 cells transfected with pDsRed2-N1 using the DuCV-positive sera (Fig. 2a,b).

The H1299 cells were washed, mounted, and analyzed by fluorescence microscopy (AF6000, Leica, Wetzlar, Germany) at $48 \mathrm{hr}$ after transfection. The results showed that DsRed2tagged Reps (Rep1-DsRed2 and Rep2-DsRed2) were observed exclusively in the nuclei of transfected H1299 cells,
Table 1. The primers used in this study

\begin{tabular}{|c|c|}
\hline Primers & Oligonucleotide sequence in $5^{\prime}-3$ 'direction \\
\hline Rep1-F & $\mathrm{CAG}$ \\
\hline Rep1-R & te \\
\hline Rep2-F & GAATTCGCAGGATGGCGAAGAGCG \\
\hline Rep2-R & $\begin{array}{l}\text { GATCCTGGTAGTTTATTGGGAACG } \\
\text { GGTA }\end{array}$ \\
\hline Rep1- $\Delta 10-37$ F & $\begin{array}{l}\text { TGGCAACTACTCCTACTTCG } \\
\text { CGGA }\end{array}$ \\
\hline Rep1- $\Delta 10-37$ R & $\begin{array}{l}\text { GATTGCGAAGTAGGAGTAGTT } \\
\text { TTA }\end{array}$ \\
\hline Rep1- $\Delta 244-274$ F & AAGACGTTGATCGTTGACAAGTAT \\
\hline Rep1- $\Delta 244-274$ R & $\begin{array}{l}\text { GT TCATACT TGTCA ACGAT } \\
\text { TTAGCAAC }\end{array}$ \\
\hline Rep2- $\Delta$ N10-3 & $\begin{array}{l}\text { G A A G A G C G G C A A C TA C T C ATA C T T T } \\
\text { GCTATCGTCGGCGAG }\end{array}$ \\
\hline Rep2- $\Delta$ N10-37 R & $\begin{array}{l}\text { CTCGCCGACGATAGCAAAGTATGAGTAGTT } \\
\text { GCCGCTCTTC }\end{array}$ \\
\hline
\end{tabular}

(a)

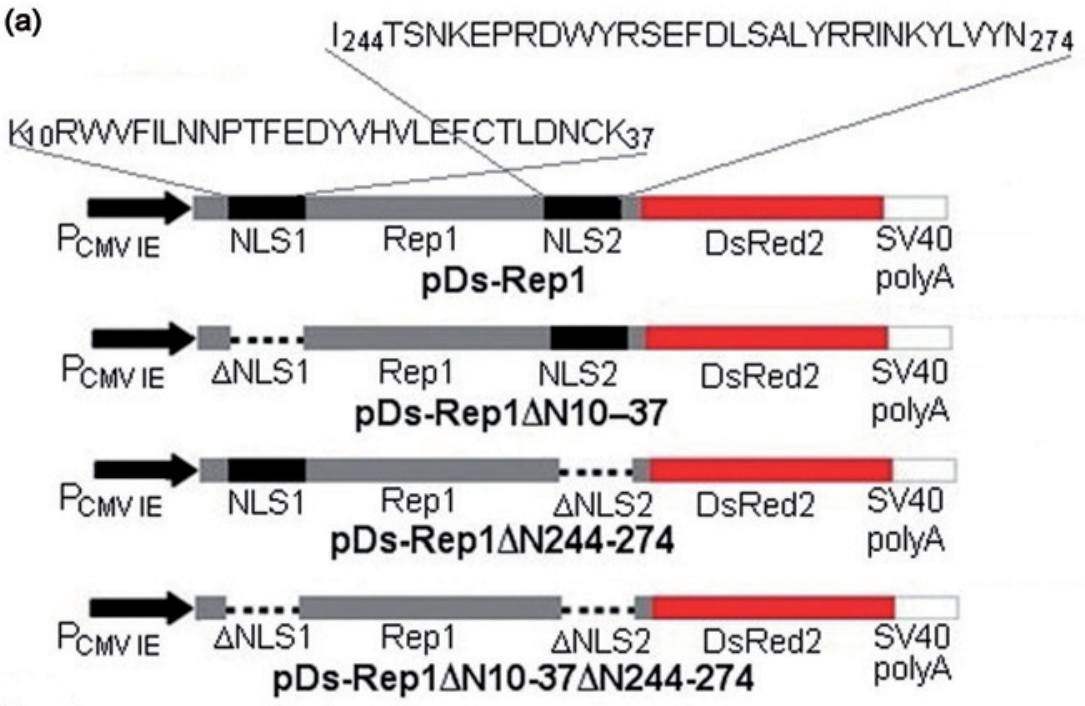

(b)

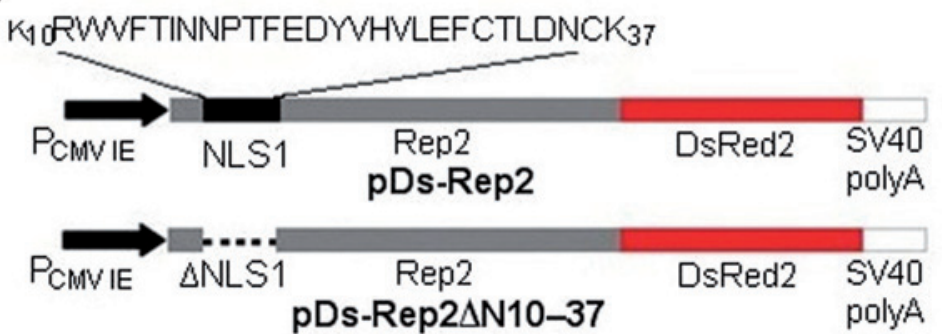

Fig. 1

A schematic representation of the replication protein (Rep) derivatives of DuCV used in this study

(a) Expression vector carrying the red fluorescent protein (DsRed2)-tagged truncated recombinant DuCV Rep1 proteins. (b) Expression vector carrying the DsRed2-tagged truncated recombinant DuCV Rep2 proteins. The human cytomegalovirus (CMV) immediate early promoter (black arrows) was fused to the $\mathrm{N}$ termini of the Reps (gray boxes). The regions of the Reps corresponding to their putative NLSs are indicated by thick black lines. Residues constituting the potential NLSs are indicated. Dashed black lines represent the deleted regions in each of the truncated derivatives. Thick red lines represent the DsRed2 protein fused to the C termini of the Reps. 
(a)

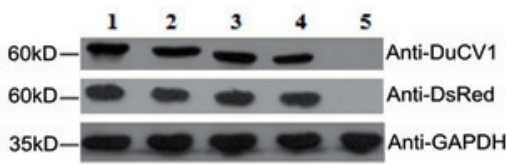

(b)

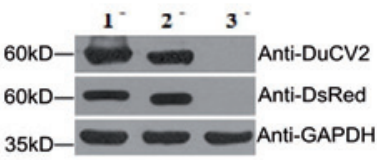

(c)

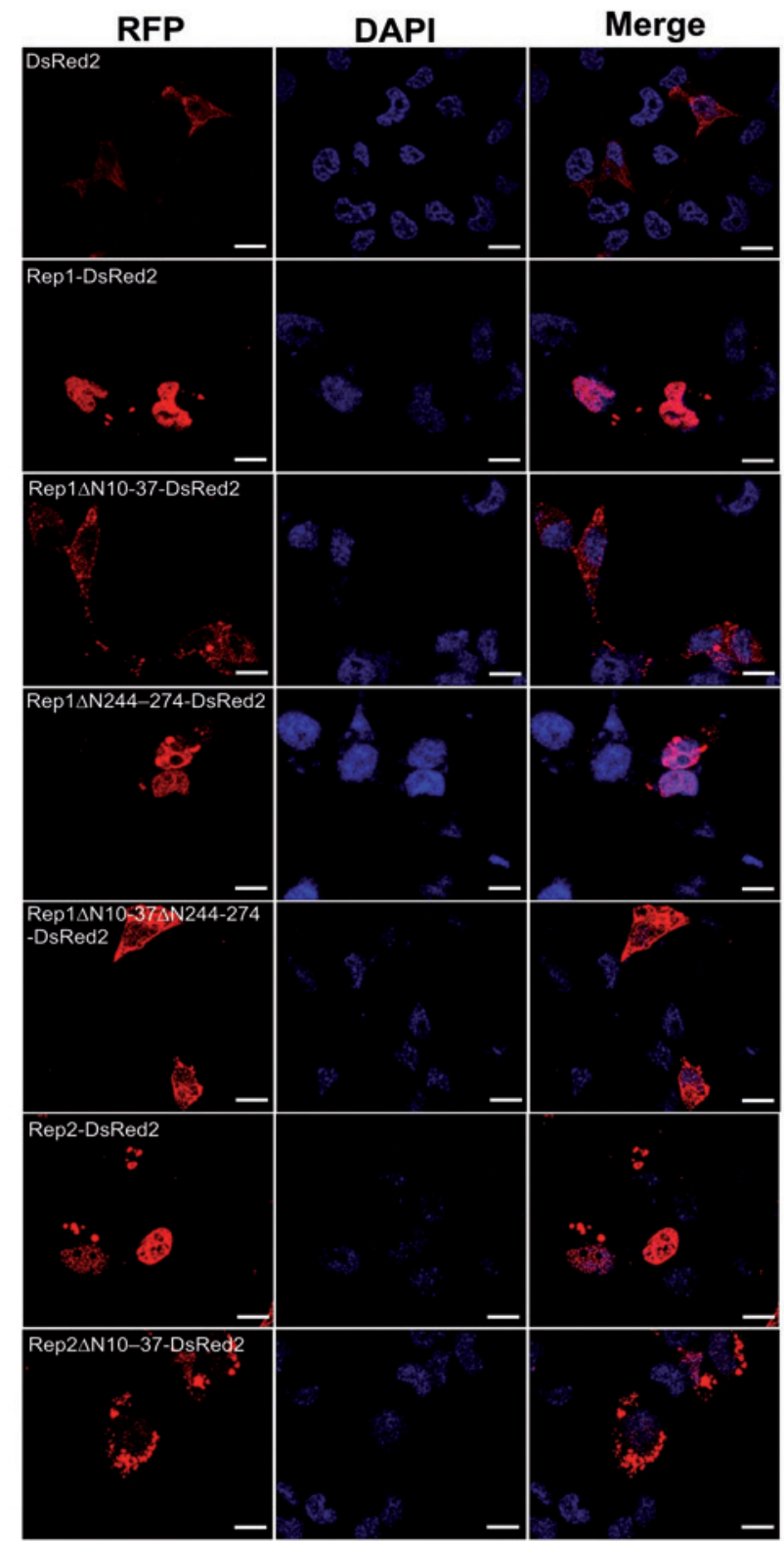

Fig. 2

Analysis of Rep expression in H1299 cells

(a) Analysis of truncated DuCV Rep1 proteins by Western blotting. Rep1-DsRed2 (lane 1), Rep1 $\Delta$ N10-37-DsRed2 (lane 2), Rep1 $\Delta$ N244-274-DsRed2 (lane 3), Rep1 $\Delta \mathrm{N} 10-37 \Delta \mathrm{N} 244-274-\mathrm{DsRed} 2$ (lane 4), and DsRed2 (lane 5). (b) Analysis of truncated DuCV Rep2 proteins by Western blotting. Rep2DsRed2 (lane 1), Rep2 $\Delta$ N10-37-DsRed2 (lane 2), and DsRed2 (lane 3). (c) Subcellular distribution of DuCV Rep1 and Rep2 proteins in H1299 cells. In the top row, DsRed2 expressed alone is distributed throughout the cytoplasm of infected cells. In the second and sixth rows, DsRed2-tagged Reps were observed exclusively in the nuclei of infected cells. In the third and seventh rows, deletion of the first putative NLS (N-terminal amino acid residues 10-37 of Rep1 and Rep2) completely abolished the translocation of the fusion proteins Rep1 $\Delta$ N10-37-DsRed2 and Rep2 $\Delta$ N10-37-DsRed2 into the nuclei of infected cells, resulting in their distribution throughout the cytoplasm, as occurred for DsRed2. In the fourth row, removal of the second putative NLS (residues 244-274) of Rep1 clearly did not impair the nuclear import of the Rep1-DsRed2 fusion protein. In the fifth row, deletion of residues 10-37 and 244-274 completely abolished the translocation of the Rep1 $\Delta$ N10-37 $\Delta$ N244-274-DsRed2 fusion protein into the nuclei of infected cells, resulting in its distribution throughout the cytoplasm, as occurred for DsRed2. 
and fluorescence was generally distributed evenly throughout the nuclear region, while DsRed 2 was distributed throughout the cytoplasm of transfected cells (Fig. 2c). Similarly to the Rep1-DsRed2 proteins, the Rep1 $\Delta$ N244-274-DsRed2 fusion protein was distributed exclusively in the nuclei of transfected H1299 cells (Fig. 2c), thus the deletion of the putative NLS located at residues 244-274 of Rep1 did not significantly alter its subcellular localization. In contrast, the Rep fusion proteins Rep1 $\Delta$ N10-37-DsRed2, Rep2 $\Delta$ N10-37-DsRed2, and Rep1 $\Delta$ N10-37 $\Delta$ N244-274-DsRed2 were uniformly distributed throughout the cytoplasm of the transfected H1299 cells, as was DsRed2, which indicated that deleting N-terminal residues 10-37 of Rep 1 and Rep 2 completely abrogated the nuclear translocation of their respective fusion proteins (Fig. 2c). The results showed that only the NLS located at residues 10-37 of Rep1 and Rep2 had nuclear targeting activity.

The Rep protein is the viral replication-associated protein of DuCV (Hattermann et al., 2003). To date, we have very little knowledge about the functional role of this protein in the replication and maturation of DuCV. In this study, the proteins Rep 1 and Rep2 of DuCV were successfully expressed in non-small cell lung cancer cells. A western blot assay showed that Rep1 and Rep2 were detected by DuCV-positive serum (Fig. 2a,b), which indicated that the Reps of DuCV are in fact expressed in ducks infected with DuCV.

Two potential NLSs are located at the N- and C-termini of DuCV Rep1, whereas only one potential NLS is located at the N-terminus of DuCV Rep2 (Fig. 1). To test whether these potential NLSs were functional, we constructed a series of plasmids by sequentially deleting the N-terminal and Cterminal regions between amino acid residues $10-37$ and 244-274 of Rep1 and Rep2, respectively. The results indicated that deleting the N-terminal NLSs (residues 10-37) of Rep1 and Rep2 clearly abrogated nuclear translocation, while deleting the putative C-terminal NLS located at residues 244-274 of Rep1 did not significantly alter its subcellular localization, confirming that the karyophilic activity of these proteins was associated with their N-terminal NLSs. The data also suggested that the nuclear translocation mechanism of DuCV Rep1 might be the same as that of DuCV Rep2.

Many different viruses have been shown to either target nuclear proteins for use in virus infection, and viral proteins have been shown to localize to the nucleus. Although Rep is required to initialize viral DNA replication, the continuation of the process is dependent upon cellular enzymes expressed during the cell's S phase, and it commences only after the host cell has passed through mitosis (Tischer et al., 1987). The Rep protein of the beak feather disease virus, when expressed alone, is restricted to the cytoplasm of insect cells, whereas it localizes to the nucleus when co-expressed with the CP (Heath et al., 2006). In a stark contrast, there are three NLSs with nuclear targeting activity that are located at the identical N-termini of
Rep and Rep' of PCV (Finsterbusch et al., 2005). In this study, the Rep proteins of two DuCV genotypes actively localized to the nucleus when they were expressed in non-small cell lung cancer cells (Fig. 2c). The data presented in this study and in our previous study (Xiang et al., 2013) showed that the CP and Reps each have one NLS at their N-terminus, which indicates that these proteins might independently translocate into the nucleus during viral replication.

Acknowledgements. This study was funded by grants from the Shandong Provincial Natural Science Foundation, China (ZR2013CM009), and the Shandong Modern Agricultural Technology \& Industry System, China (SDAIT-13-011-15).

\section{References}

Cheung AK (2004): Identification of the essential and non-essential transcription units for protein synthesis, DNA replication and infectious virus production of porcine circovirus type 1. Arch. Virol. 149, 975-988. http://dx.doi.org/10.1007/ s00705-003-0249-8

Cheung AK (2003): The essential and nonessential transcription units for viral protein synthesis and DNA replication of porcine circovirus type 2. Virology 313, 452-459. http:// dx.doi.org/10.1016/S0042-6822(03)00373-8

Cheung AK (2006): Rolling-circle replication of an animal circovirus genome in a theta-replicating bacterial plasmid in Escherichia coli. J. Virol. 80, 8686-8694. http://dx.doi. org/10.1128/JVI.00655-06

Cheung AK (2007): A stem-loop structure, sequence non-specific, at the origin of DNA replication of porcine circovirus is essential for termination but not for initiation of rollingcircle DNA replication. Virology 363, 229-235. http:// dx.doi.org/10.1016/j.virol.2007.01.017

Finsterbusch T, Steinfeldt T, Caliskan R, Mankertz A (2005): Analysis of the subcellular localization of the proteins Rep, Rep and Cap of porcine circovirus type 1. Virology 343, 36-46. http://dx.doi.org/10.1016/j.virol.2005.08.021

Hattermann K, Schmmitt C, Soike D, Mankertz A (2003): Cloning and sequencing of Duck circovirus (DuCV). Arch. Virol. 148, 2471-2480. http://dx.doi.org/10.1007/s00705-003 -0181-y

Heath L, Williamson AL, Rybicki EP (2006): The capsid protein of beak and feather disease virus binds to the viral DNA and is responsible for transporting the replication-associated protein into the nucleus. J. Virol. 80, 7219-7225. http:// dx.doi.org/10.1128/JVI.02559-05

Jiang S, Zhang X, Liu S, Wang Y, Kong Y, Wei X, Sun Y, Zhao Q (2008): PCR Detection and Sequence Analysis of Duck Circovirus in Sick Muscovy Ducks. Virol. Sin. 23, 265271. http://dx.doi.org/10.1007/s12250-008-2934-y

Li Z, Wang X, Zhang R, Chen J, Xia L, Lin S, Xie Z, Jiang S (2014): Evidence of possible vertical transmission of duck circovirus. Vet. Microbiol. 174, 229-232. http://dx.doi. org/10.1016/j.vetmic.2014.09.001 
Liu Q, Tikoo SK, Babiuk LA (2001): Nuclear localization of the ORF2 protein encoded by porcine circovirus type 2. Virology 285, 91-99. http://dx.doi.org/10.1006/ viro.2001.0922

Liu SN, Yang JB, Zhang XX, Chen Z, Kong YB, Zhu YL, Jiang SJ (2010a): Prokaryotic expression and antiserum preparation of replication protein of duck circovirus FJ0601 strain. China J. Vet. Sci. 30, 10-12, 18 (in Chinese).

Liu SN, Zhang XX, Zou JF, Xie ZJ, Zhu YL, Zhao Q, Zhou EM., Jiang SJ (2010b): Development of an indirect ELISA for the detection of duck circovirus infection in duck flocks. Vet. Microbiol. 145, 41-46. http://dx.doi.org/10.1016/j. vetmic.2010.03.010

Mankertz A, Hillenbrand B (2001): Replication of porcine circovirus type 1 requires two proteins encoded by the viral rep gene. Virology 279, 429-438. http://dx.doi.org/10.1006/ viro. 2000.0730

Mankertz A, Mueller B, Steinfeldt T, Schmitt, C, Finsterbusch T (2003): New reporter gene-based replication assay reveals exchangeability of replication factors of porcine circovirus types 1 and 2. J. Virol. 77, 9885-9893. http://dx.doi. org/10.1128/JVI.77.18.9885-9893.2003

Meerts P, Misinzo G, McNeilly F, Nauwynck HJ (2005): Replication kinetics of different porcine circovirus 2 strains in PK-15 cells, fetal cardiomyocytes and macrophages. Arch. Virol. 150, 427-441. http://dx.doi.org/10.1007/ s00705-004-0444-2

Shuai JB, Wei W, Li XL, Chen N, Fang WH (2008): Mapping of the nuclear localization signals of porcine circovirus type 1 ORF2 protein. Acta Biochim. Biophis. Sin. 40, 71-77. http://dx.doi.org/10.1111/j.1745-7270.2008.00377.x

Soike D, Albrecht K, Hattermann K., Schmitt C, Mankertz A (2004): Novel circovirus in mulard ducks with developmental and feathering disorders. Vet. Rec. 154, 792-793. http:// dx.doi.org/10.1136/vr.154.25.792
Steinfeldt T, Finsterbusch T, Mankertz A (2001): Rep and Rep' protein of porcine circovirus type 1 bind to the origin of replication in vitro. Virology 291, 152-160. http://dx.doi. org/10.1006/viro.2001.1203

Steinfeldt T, Finsterbusch T, Mankertz A (2006): Demonstration of nicking/joining activity at the origin of DNA replication associated with the rep and rep' proteins of porcine circovirus type 1. J. Virol. 80, 6225-6234. http://dx.doi. org/10.1128/JVI.02506-05

Tischer I, Peters D, Rasch R, Pociuli S (1987): Replication of porcine circovirus: induction by glucosamine and cell cycle dependence. Arch. Virol. 96, 39-57. http://dx.doi. org/10.1007/BF01310989

Wang D, Xie X, Zhang D, Ma G, Wang X, Zhang D (2011): Detection of duck circovirus in China: A proposal on genotype classification. Vet. Microbiol. 147, 410-415. http://dx.doi. org/10.1016/j.vetmic.2010.07.014

Xiang QW, Wang X, Xie ZJ, Sun YN, Zhu YL, Wang SJ, Liu HJ, Jiang SJ (2012): ORF3 of duck circovirus: a novel protein with apoptotic activity. Vet. Microbiol. 159, 251-256. http:// dx.doi.org/10.1016/j.vetmic.2012.03.045

Xiang QW, Zou JF, Wang X, Sun YN, Gao JM, Xie ZJ, Wang Y, Zhu YL, Jiang SJ (2013): Identification of two functional nuclear localization signals in the capsid protein of duck circovirus. Virology 436, 112-117. http://dx.doi. org/10.1016/j.virol.2012.10.035

Zhang X, Jiang S, Wu J, Zhao Q, Sun Y, Kong Y, Li X, Yao M, Chai T (2009): An investigation of duck circovirus and co-infection in Cherry Valley ducks in Shandong Province, China. Vet. Microbiol. 133, 252-256. http://dx.doi. org/10.1016/j.vetmic.2008.07.005

Zhang XX, Liu SN, Xie ZJ, Kong YB, Jiang SJ (2012): Complete genome sequence analysis of duck circovirus strains from Cherry Valley duck. Virol. Sin. 27, 154-164. http://dx.doi. org/10.1007/s12250-012-3214-4 